\title{
Precoder Design and Power Allocation for MIMO Cognitive Radio Two-Way Relaying Systems
}

\author{
Lokman Sboui*, Hakim Ghazzai ${ }^{\S}$, Zouheir Rezki*, and Mohamed-Slim Alouini* \\ * King Abdullah University of Science and Technology (KAUST), \\ Computer, Electrical and Mathematical Science and Engineering Division (CEMSE), \\ Thuwal 23955-6900, Saudi Arabia. \\ Email: \{lokman.sboui, zouheir.rezki, slim.alouini\}@kaust.edu.sa

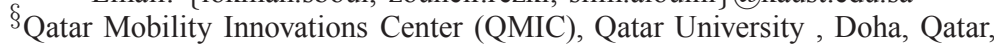 \\ Email: hakimg@qmic.com
}

\begin{abstract}
In this paper, we study a multiple-antenna two-way relaying (TWR) cognitive radio (CR) system. A space alignment (SA) technique is adopted by the secondary users (SUs) to avoid interference with the primary users (PUs). We derive the optimal power allocation that maximizes the TWR achievable SU sumrate while respecting the total power budget and the relay power constraints. We also analyze the case in which the relay is able to optimize its gain matrix structure to enhance the SU sum-rate. In the numerical results, we quantify the sum-rate gain of using the SA in the TWR CR and we show that the SU sum-rate is very limited when the relay power is low or the PU power and its resulting interference are high. In addition, we optimize the relay gain using an iterative algorithm and compare between different relay matrix structures.
\end{abstract}

Index Terms-MIMO space alignment, cognitive radio, twoway relaying, precoder design.

\section{INTRODUCTION}

Spectrum scarcity is one of the most challenging problems when designing new wireless communication systems. In fact due to the spectrum shortage reported by the FCC [2], new efficient band allocation policies have to be adopted. In this context, the cognitive radio (CR) paradigm was introduced to overcome this issue [3]-[5]. In this paradigm, secondary users (SUs) share the spectrum of licensed primary users (PUs) under the condition of not affecting the primary communication. Recently, many techniques were presented to enhance CR communications, e.g., relaying and multiple-input multipleoutput (MIMO) communication systems. MIMO communications are based on multiple antennas to enhance the spectral efficiency [6]. In CR MIMO technique, the space alignment (SA) was introduced to enhance the cognitive throughput [7][11]. In relaying systems, additional nodes are retransmitting the received signal to the destination in the aim of reducing the communication cost and enhancing reliability [12]-[14]. Relaying is very efficient when there is no direct line of sight between the transmitter and the receiver, e.g., cell edge case. Relaying can be based on one of the following techniques: i) amplify-and-forward (AF) [15], [16]; the relay amplifies the received signal and sends it to the destination, ii) decode-andforward (DF) [17]; the relay decodes the signal, re-encodes

A part of this work has been presented at the 2015 IEEE International Conference on Communications (ICC'15), London, UK, June 2015 [1]. it and sends it, and iii) compress-and-forward (CF) [18]; the relay compresses the received signal and forwards an estimation of it. In the traditional relaying, known also as one-way [19] (OWR), the relay receives the signal in one time slot and sends it to the destination in the second time slot. Consequently, having two transceivers from both sides, transmission requires four time slots to be accomplished. Recently, the two-way relaying [20]-[22] (TWR) has been proposed as an efficient way to accomplish the transmission in two time slots only [23], [24]. In the first time slot, the relay receives two signals from the communication terminals. In the second time slot, the relay broadcasts the mixture of the two signals. Consequently, each terminal can decode the intended message by performing a self interference cancellation on the total signal. In this case, the two transmissions from both sides will only require two time slots offering a higher spectral efficiency than the OWR. The MIMO OWR CR capacity was studied in [13], [14]. Authors in [25]-[27] considered cognitive AF TWR and used beamforming design on the SU to avoid the interference with the PU. In [25], the relay is used to assist the PU communications as well. The TWR CR power allocation was studied in [28], [29] and in [21], where the authors analyzed the cognitive performance of an AF TWR in a spectrum sensing framework. In [28], the authors determined the optimal power for the SU which is acting as a DF twoway relay and focused on the outage performance. In order to better enhance the capacity, few studies focused on the relay beamforming in the TWR-MIMO framework [16], [29], [30]. In [31], optimal relay matrix was computed for a MIMO relay and the corresponding rate region was presented. In the CR framework, the authors in [32] presented precoder design when the channel state information (CSI) is not perfect with SISO PU and MIMO SU. In [27], the authors presented many relay strategies to maximize the SU sum-rate while respecting PU rate requirements.

To the best of our knowledge, the SA technique in cognitive TWR MIMO was not studied before. In this paper, we propose an efficient solution for CR TWR networks in which the SUs employ the SA technique in order to accomplish their bi-directional communication without affecting the primary transmission. In our work, we design a precoding scheme at secondary sources and relays such that the secondary communication becomes transparent to PUs. We study the 
power allocation problem of the SUs in a TWR scenario similarly to [21] but in a cognitive framework where the spectrum sensing is no more needed. However, the terminals still need to perform channel sensing for the CSI acquisition. Meanwhile, we use the SA technique to avoid interfering with the PUs that are performing a direct two-way communication. Note that, in [21], the authors studied a SISO system while, in our work, we study the MIMO case which is a more general setting. The main contributions of this paper are summarized as follows:

- We design the decoders, at the secondary terminals and at the relay, which allow the secondary transmission to be totally transparent to the PUs on a MIMO TWR system using the SA technique;

- We derive a closed-form expression of the optimal power allocation per antenna for each secondary terminal;

- We propose to optimize further the relay matrix, along with optimizing the power levels, to maximize the SU rate. We present three possible structures of the relay matrix depending on their complexity-efficiency tradeoff;

- We quantify, in the numerical results, the effect of the relay parameters (power and matrix gain) on the SU sum-rate. We also perform comparison between different relay matrix complexity structures and highlight the corresponding performances.

The rest of this paper is organized as follows. In Section II, the CR TWR system model is described. Section III presents the PU power allocation design. The TWR SU optimal power allocations for a fixed relay gain are derived in Section IV. The relay gain optimization is described in Section V. Numerical results are discussed in Section VI. Finally, the paper is concluded in Section VII.

\section{System Model}

We consider a cognitive radio network depicted by Fig. 1, consisting of one pair of PU transceivers, denoted by $P_{1}$ and $P_{2}$, and one pair of $\mathrm{SU}$ transceivers denoted by $S_{1}$ and $S_{2}$. Two-way communication based on time division duplex (TDD) is performed in the primary link while the SUs exchange their messages using an AF two-way relaying technique. A relay $(\mathrm{R})$ is introduced to ensure the communication between the secondary terminals by amplifying and forwarding the received secondary signals. The PUs, as licensed users, exploit the total bandwidth while the SUs, as unlicensed users, are allowed to share opportunistically the spectrum and to access the channel without affecting the primary communication. Each primary and secondary node is equipped with $N_{p}$ and $N_{s}$ antennas, respectively. We denote by $\boldsymbol{H}_{p}, \boldsymbol{H}_{s_{1}}, \boldsymbol{H}_{s_{2}}, \boldsymbol{H}_{s_{1} p_{2}}, \boldsymbol{H}_{s_{2} p_{2}}, \boldsymbol{H}_{p_{1} R}$ the channel gain matrices representing the links between $P_{1}$ and $P_{2}, S_{1}$ and $R$, $S_{2}$ and $R, P_{2}$ and $S_{1}, P_{2}$ and $S_{2}$, and $P_{1}$ and $R$, respectively. All the channel gains are assumed to be independent and constant during two consecutive time slots. In our framework, where the objective is to investigate the performance of this novel SA-based solution for CR TWR, we assume that full channel state information (CSI) is available at all terminals using MMSE approach [33]. That is, each transmitter sends

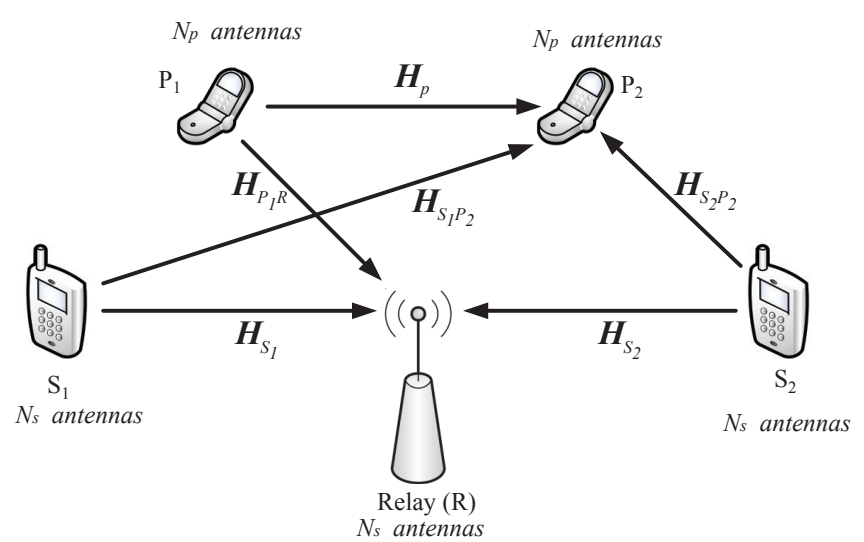

(a) First time slot transmissions: The senders are $P_{1}, S_{1}$ and $S_{2}$, whereas $P_{2}$ and $R$ are listeners.

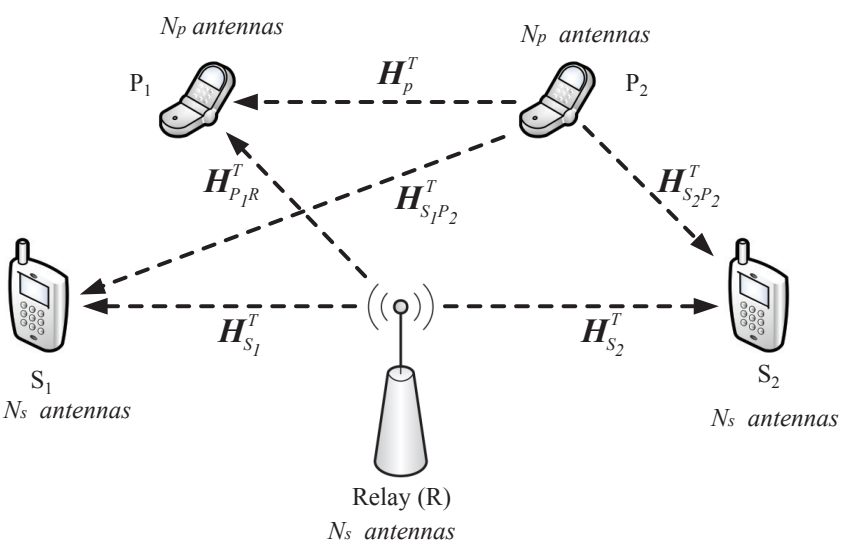

(b) Second time slot transmissions: The senders are $P_{2}$ and $R$ whereas $P_{1}, S_{1}$ and $S_{2}$ are listeners.

Figure 1: Two way relaying cognitive radio system model.

pilots and the corresponding receivers estimate the channel and broadcast their estimate to the whole network, so that each terminal is aware of an estimate of all channel involved in the network. In this work, we neglect the estimation error and assume that the estimates are very close to the actual channel gains.

The transmissions between primary transceivers is performed during two time slots. In addition, the SUs synchronize their transmissions within the primary time slots as well. Hence, the secondary transmission is completely transparent to the PUs. During the first time slot, $S_{1}, S_{2}$ and $P_{1}$ are transmitting while $R$ and $P_{2}$ are listening. We denote by $\boldsymbol{y}_{p_{2}}$ and $\boldsymbol{y}_{\boldsymbol{R}}$ the received signals at $P_{2}$ and $R$, respectively. In this first time slot, the relay perform time synchronization by estimating the channel impairments caused by the timing offset and the difference in channel gains using maximum likelihood algorithm presented in [34].

During the second time slot, $P_{2}$ transmits its signal to $P_{1}$ while the relay amplifies the received secondary signal through a gain matrix denoted by $W$ and forwards it to $S_{1}$ and $S_{2}$. We denote by $\boldsymbol{y}_{p_{1}}, \boldsymbol{y}_{s_{1}}$ and $\boldsymbol{y}_{s_{2}}$ the received signals at $P_{1}, S_{1}$, and $S_{2}$, respectively. The expressions of the received signals at the two time slots are given as follows 


\section{Received signals at the first time slot}

$$
\begin{aligned}
& y_{p_{2}}=H_{p} \Phi_{p_{1}} x_{p_{1}}+H_{s_{1} p_{2}} \Phi_{s_{1}} x_{s_{1}}+H_{s_{2} p_{2}} \Phi_{s_{2}} x_{s_{2}}+n_{p_{2}} \\
& y_{R}=H_{s_{1}} \Phi_{s_{1}} x_{s_{1}}+H_{s_{2}} \Phi_{s_{2}} x_{s_{2}}+H_{p_{1} R} \Phi_{p_{1}} x_{p_{1}}+n_{R}
\end{aligned}
$$

\section{Received signals at the second time slot}

$$
\begin{aligned}
& \boldsymbol{y}_{p_{1}}=\boldsymbol{H}_{p}^{T} \boldsymbol{\Phi}_{\boldsymbol{p}_{\mathbf{2}}} \boldsymbol{x}_{\boldsymbol{p}_{\mathbf{2}}}+\boldsymbol{H}_{p_{1} R}^{T} \boldsymbol{W} \boldsymbol{y}_{\boldsymbol{R}}+\boldsymbol{n}_{\boldsymbol{p}_{\mathbf{1}}}, \\
& \boldsymbol{y}_{s_{1}}=\boldsymbol{H}_{s_{1}}^{T} \boldsymbol{W} y_{R}+H_{s_{1} p_{2}}^{T} \Phi_{p_{2}} x_{p_{2}}+n_{s_{1}} \text {, } \\
& y_{s_{2}}=H_{s_{2}}^{T} W y_{R}+H_{s_{2} p_{2}}^{T} \Phi_{p_{2}} x_{p_{2}}+n_{s_{2}},
\end{aligned}
$$

where $\boldsymbol{\Phi}_{\boldsymbol{i}}, i \in\left\{p_{1}, p_{2}, s_{1}, s_{2}\right\}$ are the linear precoding matrices applied to transmitter $i$. The signals $\boldsymbol{x}_{\boldsymbol{i}}$ 's are independent complex Gaussian random vectors with zero mean. We consider $\boldsymbol{P}_{\boldsymbol{i}}=\mathbb{E}\left[\boldsymbol{x}_{\boldsymbol{i}} \boldsymbol{x}_{\boldsymbol{i}}{ }^{H}\right]$ to be the covariance matrix of the vector $\boldsymbol{x}_{\boldsymbol{i}}$, where $\mathbb{E}[\cdot]$ is the expectation over all channels realizations, ${ }^{T}$ and ${ }^{H}$ designates the transpose and the Hermitian operators, respectively. The transmitter $i$ is subject to a peak power constraint $\operatorname{Tr}\left(\boldsymbol{\Phi}_{\boldsymbol{i}} \boldsymbol{P}_{\boldsymbol{i}} \boldsymbol{\Phi}_{\boldsymbol{i}}{ }^{H}\right) \leq P_{\text {tot }, i}$ where $\operatorname{Tr}(\boldsymbol{A})=\sum_{j} A(j, j)$ is the trace of the matrix $\boldsymbol{A}$, and $P_{\text {tot, } \mathrm{i}}$ is the total power budget of user $i, i \in\left\{p_{1}, p_{2}, s_{1}, s_{2}\right\}$. The power of the relay $R$, is limited to its power budget denoted by $P_{r}$. Finally, $\boldsymbol{n}_{\boldsymbol{i}}$ and $\boldsymbol{n}_{\boldsymbol{R}}$ indicate the zero mean additive white Gaussian noise (AWGN) vector at the node $i$ and the relay with an identity covariance matrix denoted by $\boldsymbol{I}_{\boldsymbol{N}_{p}}$ for the primary system and $\boldsymbol{I}_{\boldsymbol{N}_{s}}$ for the secondary system.

\section{Primary Achievable Rates}

In this section, we compute the PU achievable rate by optimizing the power allocation over PU antennas while ignoring the SU presence. In fact, the PUs act as if the SUs (i.e., secondary terminals and relay) do not exist in the network. By applying a singular value decomposition (SVD) to $\boldsymbol{H}_{\boldsymbol{p}}$, the PU, i.e., either $P_{1}$ in the first time slot or $P_{2}$ in the second time slot, transmits through parallel channels characterized by their associated eigenmodes. The SVD of the matrix is denoted by $\boldsymbol{H}_{p}=\boldsymbol{U}_{p_{1}} \boldsymbol{\Lambda}_{p} \boldsymbol{V}_{\boldsymbol{p}_{1}}{ }^{H}$ where $\boldsymbol{U}_{p_{1}}$ and $\boldsymbol{V}_{p_{1}}$ are two unitary matrices and $\Lambda_{p}$ is a diagonal matrix that contains the ordered singular values of $\boldsymbol{H}_{p}$ denoted by $\lambda_{1} \geq \lambda_{2} \geq \cdots \geq \lambda_{N}$. Similarly, we have $\boldsymbol{H}_{p}{ }^{T}=\boldsymbol{U}_{\boldsymbol{p}_{\mathbf{2}}} \boldsymbol{\Lambda}_{\boldsymbol{p}} \boldsymbol{V}_{\boldsymbol{p}_{\mathbf{2}}}{ }^{H}$ where $\boldsymbol{U}_{\boldsymbol{p}_{\mathbf{2}}}$ and $V_{p_{2}}$ are two unitary matrices. Note that the singular values are the same for $\boldsymbol{H}_{p}$ and $\boldsymbol{H}_{p}{ }^{T}$. To transform the PU MIMO relay channel to $N$ parallel channels, we employ the linear precoding $\Phi_{p_{1}}$ and $\Phi_{p_{2}}$ and the decoding $\Psi_{p_{1}}$ and $\Psi_{p_{2}}$ at the $P_{1}$ node and $P_{2}$ node, respectively, as follows

$$
\Phi_{p_{1}}=V_{p_{1}}, \Phi_{p_{2}}=V_{p_{2}}, \Psi_{p_{1}}=U_{p_{1}} \text { and } \Psi_{p_{2}}=U_{p_{2}} .
$$

In our CR setting, the secondary interference at PUs will be received in an orthogonal space to the PU transmit space. Thus, the SU activity is transparent and does not affect the PU quality of service (QoS). The output received signal at each PU node after decoding becomes

$$
\begin{aligned}
& r_{p_{1}}=\Psi_{p_{1}}^{H} y_{p_{1}}=\Lambda_{p} x_{p_{2}}+U_{p_{1}}^{H} n_{p_{1}}, \\
& r_{p_{2}}=\Psi_{p_{2}}^{H} y_{p_{2}}=\Lambda_{p} x_{p_{1}}+U_{p_{2}}^{H} n_{p_{2}} .
\end{aligned}
$$

In order to maximize their rates, the PUs proceed with optimizing their power allocation over the $N_{p}$ parallel antennas by solving the following optimization problem

$$
\begin{aligned}
& \max _{\boldsymbol{P}_{\boldsymbol{p}}} R_{p}=\sum_{j=1}^{N_{p}} \log _{2}\left(1+P_{p}(j, j) \lambda_{j}^{2}\right), \\
& \text { s.t. } \operatorname{Tr}\left(\boldsymbol{P}_{\boldsymbol{p}}\right) \leq P_{\text {tot, } \mathrm{p}},
\end{aligned}
$$

where $p$ stands for $p_{1}$ or $p_{2}$ depending on the considered transmitter. Note that the achievable rates for both $P_{1}$ and $P_{2}$ are identical since the channel gain remains constant in two consecutive time slots. Thus, both users have the same optimal power profile. The optimal power is given, $\forall j=1, \ldots, N_{p}$, by

$$
P_{p}^{*}(j, j)=\left[\frac{1}{\mu_{p}}-\frac{1}{\lambda_{j}^{2}}\right]^{+},
$$

where $[\cdot]^{+}=\max (0, \cdot)$ and $\mu_{p}$ is the Lagrange multiplier corresponding to the primary total power constraint expressed in (10). From (11), when the channel gain is poor, i.e., $\lambda_{j}$ 's have small values, we note that the number of used eigenmodes by PUs can be less than the total number of antennas $N_{p}$. This case occurs when the optimal power allocated to the $j^{\text {th }}$ antenna is zero (i.e., $P_{p}^{*}(j, j)=0$ ). Consequently, the SUs can freely exploit the unused eigenmodes. We denote by $n_{p}$ $\left(0 \leq n_{p}<N_{p}\right)$ the number of unused primary eigenmodes. Note that if we have $N_{p}-n_{p}+1>N_{s}$ then there is no free eigenmodes to be used by the secondary system. Then, we distinguish two sets of eigenmodes: $N_{p}-n_{p}$ eigenmodes used by the PUs and $n_{p}$ unused eigenmodes from which $n$ eigenmodes can be freely exploited by the SUs where $n=\min \left\{n_{p},\left[N_{s}-N_{p}+n_{p}\right]^{+}\right\}$.

\section{Secondary Achievable Rates and Space ALIGNMENT PRECODING}

This section introduces the proposed linear precoding and decoding matrices used to maximize the SU sum-rate without affecting the PU rates. The proposed scheme is employed to exploit the SA technique, presented in [7], which allows the SUs to freely exploit the unused primary eigenmodes. Note that the secondary system is able to determine the free eigenmodes by only knowing the PUs channel $\boldsymbol{H}_{p}$ then determine its SVD and the PUs power allocation locally. However, in the case where determining the free eigenmodes is not possible due to imperfect channel state information, one can rely, e.g., on sensing the free space similarly to [10]. From another side, we mentioned earlier that the SU transmission should be transparent to the PU. Consequently, the SUs need to limit their interference in the free PU space. That is, the $S_{1}$ and $S_{2}$ transmission should be transparent in the first time slot and the relay's transmission should be transparent in the second time slot. For this purpose, we propose a precoding scheme at the SUs terminals that considers the channel gain of their links with the PUs terminals as follows

$$
\Phi_{s_{1}}=\left(\boldsymbol{U}_{p_{1}}^{H} H_{s_{1} p_{2}}\right)^{+} \overline{\boldsymbol{P}}_{\boldsymbol{p}} \text { and } \boldsymbol{\Phi}_{s_{2}}=\left(\boldsymbol{U}_{\boldsymbol{p}_{2}}^{\boldsymbol{H}} \boldsymbol{H}_{\boldsymbol{s}_{2} \boldsymbol{p}_{2}}\right)^{+} \overline{\boldsymbol{P}}_{\boldsymbol{p}},
$$


where $(\cdot)^{+}$denotes the pseudo-inverse operator defined as $\boldsymbol{A}^{+}=\left(\boldsymbol{A}^{H} \boldsymbol{A}\right)^{+} \boldsymbol{A}^{H}$, where $\overline{\boldsymbol{P}}_{\boldsymbol{p}}$ is an $N_{p} \times N_{s}$ matrix that models the unused primary eigenmodes and is given by

$$
\bar{P}_{p}(j, j)= \begin{cases}1, \text { if } P_{p}^{*}(j, j)=0, & \text { for } j=1, \ldots, \min \left\{N_{p}, N_{s}\right\} . \\ 0, \text { otherwise, } & \end{cases}
$$

In the second time slot, by re-writing equations (4) and (5), the received signals in the secondary nodes $S_{1}$ and $S_{2}$ can be rewritten as follows

$$
\begin{aligned}
& y_{s_{1}}= \underbrace{H_{s_{1}}^{T} W H_{s_{2}} \Phi_{s_{2}} x_{s_{2}}}_{\text {S2 signal }}+\underbrace{H_{s_{1}}^{T} W H_{s_{1}} \Phi_{s_{1}} x_{s_{1}}}_{\text {Self interference }} \\
&+\underbrace{H_{s_{1}}^{T} W H_{p_{1} R} \Phi_{p_{1}} x_{p_{1}}}_{\text {Amplified } P_{1} \text { interference }}+\underbrace{H_{s_{1} p_{2}}^{T} \Phi_{p_{2}} x_{p_{2}}}_{\text {P2 interference }} \\
&+\underbrace{H_{s_{1}}^{T} W n_{R}}_{\text {Amplified noise }}+\underbrace{n_{s_{1}}}_{\text {Noise }}, \\
& y_{s_{2}}=\underbrace{H_{s_{2}}^{T} W H_{s_{1}} \Phi_{s_{1}} x_{s_{1}}}_{\text {S1 signal }}+\underbrace{H_{s_{2}}^{T} W H_{s_{2}} \Phi_{s_{2}} x_{s_{2}}}_{\text {Self interference }} \\
&+\underbrace{H_{s_{2}}^{T} W H_{p_{1} R} \Phi_{p_{1}} x_{p_{1}}}_{\text {Amplified } P_{1} \text { interference }}+\underbrace{H_{s_{2} p_{2}}^{T} \Phi_{p_{2}} x_{p_{2}}}_{\text {P2 interference }} \\
&+\underbrace{H_{s_{2}}^{T} W n_{R}}_{\text {Amplified noise }}+\underbrace{n_{s_{2}}}_{\text {Noise }} .
\end{aligned}
$$

Using their CSI, the secondary terminals can remove the self interference by eliminating their own signals. In case of imperfect CSI, self-interference can still be applied, however, it will introduce an error related to the channel estimation that can be considered as the noise. On the other hand, the received secondary signals are subject to an amplified interference generated by $P_{1}$ in addition to an interference caused by $P_{2}$ which are treated as noise. We denote by $\mathcal{M}_{N_{p} ; N_{s} ; n_{p}}$ the set of $N_{p} \times N_{s}$ matrices whose $i$-th columns are non-zero where $i=N_{p}-n+1, \cdots, \min \left\{N_{p}, N_{s}\right\}$. We can easily verify that for any $N_{p} \times N_{s}$ matrix $\boldsymbol{A}$ and $\forall M \in \mathcal{M}_{N_{p} ; N_{s} ; n_{p}}$, we have

$$
\boldsymbol{A} \times \boldsymbol{M} \in \mathcal{M}_{N_{p} ; N_{s} ; n_{p}} .
$$

In the relay side and by using (3), we perform a similar precoding on the relay matrix as follows

$$
\boldsymbol{W}=\left(\boldsymbol{U}_{\boldsymbol{p}_{1}}^{\boldsymbol{H}} \boldsymbol{H}_{\boldsymbol{p}_{1} \boldsymbol{R}}^{\boldsymbol{T}}\right)^{+} \overline{\boldsymbol{P}}_{\boldsymbol{w}} .
$$

where $\overline{\boldsymbol{P}}_{\boldsymbol{w}}$ is a matrix that belongs to $\mathcal{M}_{N_{p} ; N_{s} ; n_{p}}$. We denote by $\boldsymbol{H}_{\boldsymbol{s}_{i}}^{\text {eqv }}, i \in\{1,2\}$ the equivalent channel from the two SUs, i.e., $\boldsymbol{H}_{\boldsymbol{s}_{i}}^{\text {eqv }}=\boldsymbol{H}_{\boldsymbol{s}_{i}}^{\boldsymbol{T}} \boldsymbol{W} \boldsymbol{H}_{\boldsymbol{s}_{\bar{i}}} \boldsymbol{\Phi}_{s_{\bar{i}}}$ for $i \in\{1,2\}$ and $\bar{i}=2$ if $i=1$ and vice versa. In our case, we note that, since $\overline{\boldsymbol{P}}_{\boldsymbol{w}} \in \mathcal{M}_{N_{p} ; N_{s} ; n_{p}}$, then for $i \in\{1,2\}$, we have, from (12), $\boldsymbol{\Phi}_{s_{i}} \in \mathcal{M}_{N_{p} ; N_{s} ; n_{p}}$ which means that $\boldsymbol{H}_{\boldsymbol{s}_{\boldsymbol{i}}}^{\text {eqv }} \in \mathcal{M}_{N_{p} ; N_{s} ; n_{p}}$ as well. Consequently, the first $N_{p}-n_{p}$ signals in the signal vector $\boldsymbol{x}_{s_{i}}$ will be canceled out at the receivers. Hence, the SUs are only able to transmit only $n$ signals. Thanks to the proposed encoding schemes in (12) and (17), we ensure that the PU transmissions are interference free. Consequently, the vectors $x_{s_{i}}$ are designed such as $x_{s_{i}}(j)=0 \forall j=1, \cdots, N_{p}-n_{p}$. Now in order to design the decoders at the secondary receivers, we need to consider the form of $\boldsymbol{H}_{\boldsymbol{s}_{i}}^{\text {eqv }}$. For this, we denote by $\tilde{\boldsymbol{H}}_{\boldsymbol{s}_{i}}^{\text {eqv }}$ the matrix composed of the $n$ non-zero columns of $\boldsymbol{H}_{\boldsymbol{s}_{i}}^{\text {eqv }}$ and $\tilde{\boldsymbol{x}}_{\boldsymbol{s}_{i}}$ the $n$ non-zero values of $\boldsymbol{x}_{\boldsymbol{s}_{i}}$. We apply a decoding similar to the zero-forcing at the SU receivers as follows

$$
\boldsymbol{\Psi}_{\boldsymbol{s}_{\boldsymbol{i}}}=\left(\tilde{\boldsymbol{H}}_{\boldsymbol{s}_{\boldsymbol{i}}}^{\text {eqv }}\right)^{+}, i \in\{1,2\} .
$$

Thus, the received signal $\boldsymbol{r}_{i}=\boldsymbol{\Psi}_{s_{i}} \boldsymbol{y}_{s_{i}}, i \in\{1,2\}$ is given by:

$$
r_{s_{i}}(j)=\tilde{x}_{s_{\bar{i}}}(j)+\tilde{n}_{s_{i}}(j), j=1, \cdots, n .
$$

where $\tilde{n}_{s_{i}}=\Psi_{s_{i}} H_{s_{i}}^{T} W H_{p_{1} R} \Phi_{p_{1}} x_{p_{1}}+\Psi_{s_{i}} H_{s_{i} p_{2}} \Phi_{p_{2}} x_{p_{2}}$ $+\Psi_{s_{i}} \boldsymbol{H}_{s_{i}}^{\boldsymbol{T}} \boldsymbol{W} \boldsymbol{n}_{\boldsymbol{R}}+\boldsymbol{\Psi}_{s_{i}} \boldsymbol{n}_{s_{i}}$ is the equivalent noise at the secondary receiver $s_{i}$ with an $n$-by- $n$ covariance matrix, $\boldsymbol{Q}_{\tilde{\boldsymbol{n}}_{s_{i}}}=\mathbb{E}\left[\tilde{\boldsymbol{n}}_{\boldsymbol{s}_{i}} \tilde{\boldsymbol{n}}_{\boldsymbol{s}_{i}}^{\boldsymbol{H}}\right], i \in\{1,2\}$, given by

$$
\begin{aligned}
Q_{\tilde{n}_{s_{i}}}= & \boldsymbol{\Psi}_{s_{i}} \boldsymbol{H}_{s_{i}}^{T} W \boldsymbol{H}_{p_{1} R} V_{p_{1}} P_{p}^{*}\left(\Psi_{s_{i}} H_{s_{i}}^{T} W H_{p_{1} R} V_{p_{1}}\right)^{H} \\
& +\Psi_{s_{i}} H_{s_{i} p_{2}} V_{p_{1}} P_{p}^{*}\left(\Psi_{s_{i}} H_{s_{i} p_{2}} V_{p_{1}}\right)^{H} \\
& +\Psi_{s_{i}} H_{s_{i}}^{T} W\left(\Psi_{s_{i}} H_{s_{i}}^{T} W\right)^{H}+\Psi_{s_{i}} \Psi_{s_{i}}^{H} .
\end{aligned}
$$

Thus, the achievable rate of each secondary terminal after employing the self cancellation and the SA technique is expressed as follows

$$
R_{s_{i}}=\sum_{j=1}^{n} \log _{2}\left(1+\frac{P_{s_{i}}(j, j)}{Q_{\tilde{n}_{s_{i}}}(j, j)}\right), i \in\{1,2\},
$$

and the objective becomes to optimally allocate the power over the secondary antennas in order to maximize the total secondary sum-rate by solving the following optimization problem:

$$
\max _{\boldsymbol{P}_{s_{1}}, \boldsymbol{P}_{s_{2}}} R_{\mathrm{tot}}=R_{s_{1}}+R_{s_{2}}
$$

$$
\begin{array}{ll}
\text { s.t. } & \operatorname{Tr}\left(\boldsymbol{\Phi}_{\boldsymbol{s}_{1}} \boldsymbol{P}_{\boldsymbol{s}_{1}} \boldsymbol{\Phi}_{\boldsymbol{s}_{1}}^{\boldsymbol{H}}\right) \leq P_{\mathrm{tot}, s_{1}}, \\
& \operatorname{Tr}\left(\boldsymbol{\Phi}_{\boldsymbol{s}_{\mathbf{2}}} \boldsymbol{P}_{\boldsymbol{s}_{\mathbf{2}}} \boldsymbol{\Phi}_{\boldsymbol{s}_{\mathbf{2}}}^{\boldsymbol{H}}\right) \leq P_{\mathrm{tot}, s_{2}}, \\
& \operatorname{Tr}\left(\boldsymbol{W} \boldsymbol{H}_{\boldsymbol{s}_{1}} \boldsymbol{\Phi}_{\boldsymbol{s}_{1}} \boldsymbol{P}_{\boldsymbol{s}_{1}}\left(\boldsymbol{W} \boldsymbol{H}_{\boldsymbol{s}_{1}} \boldsymbol{\Phi}_{\boldsymbol{s}_{1}}\right)^{H}\right. \\
+ & \boldsymbol{W} \boldsymbol{H}_{\boldsymbol{s}_{\mathbf{2}}} \boldsymbol{\Phi}_{\boldsymbol{s}_{\mathbf{2}}} \boldsymbol{P}_{\boldsymbol{s}_{\mathbf{2}}}\left(\boldsymbol{W} \boldsymbol{H}_{\boldsymbol{s}_{\mathbf{2}}} \boldsymbol{\Phi}_{\boldsymbol{s}_{\mathbf{2}}}\right)^{H} \\
+ & \left.\boldsymbol{W} \boldsymbol{H}_{\boldsymbol{p}_{\mathbf{1}} \boldsymbol{R}} \boldsymbol{V}_{\boldsymbol{p}_{\mathbf{1}}} \boldsymbol{P}_{\boldsymbol{p}}^{*}\left(\boldsymbol{W} \boldsymbol{H}_{\boldsymbol{p}_{\mathbf{1}} \boldsymbol{R}} \boldsymbol{V}_{\boldsymbol{p}_{\mathbf{1}}}\right)^{H}+\boldsymbol{W} \boldsymbol{W}^{H}\right) \leq P_{R},
\end{array}
$$

where constraints (23) and (24) indicate that the sum of the allocated power over the free eigenmodes is smaller than the total power budget of $S_{1}$ and $S_{2}$, respectively, and constraint (25) indicates that the transmitted signal from the relay has to not exceed its power budget. The optimization problem (22)-(25) can be simplified using invariance of the Trace operator under the cyclic permutation. For instance, the constraint (23) can be written as $\operatorname{Tr}\left(\boldsymbol{\Phi}_{s_{1}}^{\boldsymbol{H}} \boldsymbol{\Phi}_{\boldsymbol{s}_{1}} \boldsymbol{P}_{\boldsymbol{s}_{1}}\right) \leq P_{\mathrm{tot}, s_{1}}$. By defining the matrix $\boldsymbol{A}_{\boldsymbol{s}_{1}}=\boldsymbol{\Phi}_{\boldsymbol{s}_{1}}^{\boldsymbol{H}} \boldsymbol{\Phi}_{\boldsymbol{s}_{1}}$, the constraint (23) becomes $\operatorname{Tr}\left(\boldsymbol{A}_{\boldsymbol{s}_{1}} \boldsymbol{P}_{\boldsymbol{s}_{1}}\right) \leq P_{\mathrm{tot}, s_{1}}$. Similarly, we define $\boldsymbol{A}_{s_{2}}=\boldsymbol{\Phi}_{s_{2}}^{H} \Phi_{s_{2}}$,

$\boldsymbol{B}_{\boldsymbol{s}_{1}}=\left(\boldsymbol{W} \boldsymbol{H}_{\boldsymbol{s}_{1}} \boldsymbol{\Phi}_{\boldsymbol{s}_{1}}\right)^{H} \boldsymbol{W} \boldsymbol{H}_{\boldsymbol{s}_{1}} \boldsymbol{\Phi}_{\boldsymbol{s}_{1}}$, $\boldsymbol{B}_{\boldsymbol{s}_{\mathbf{2}}}=\left(\boldsymbol{W} \boldsymbol{H}_{\boldsymbol{s}_{\mathbf{2}}} \boldsymbol{\Phi}_{\boldsymbol{s}_{\mathbf{2}}}\right)^{H} \boldsymbol{W} \boldsymbol{H}_{\boldsymbol{s}_{\mathbf{2}}} \boldsymbol{\Phi}_{\boldsymbol{s}_{\mathbf{2}}}$, $\boldsymbol{C}_{\boldsymbol{p}}=\boldsymbol{W} \boldsymbol{H}_{\boldsymbol{p}_{1} \boldsymbol{R}} \boldsymbol{V}_{\boldsymbol{p}_{1}} \boldsymbol{P}_{\boldsymbol{p}}{ }^{*}\left(\boldsymbol{W} \boldsymbol{H}_{\boldsymbol{p}_{1} \boldsymbol{R}} \boldsymbol{V}_{\boldsymbol{p}_{1}}\right)^{H}+\boldsymbol{W} \boldsymbol{W}^{H}$.

Since the constraints are linear and the objective function is concave, we use the Lagrangian method to compute the 
solution for fixed $\omega$ [35]. The Lagrangian function can be written as

$$
\begin{aligned}
\mathcal{L}= & R_{s_{1}}+R_{s_{2}}-\mu_{1}\left(\operatorname{Tr}\left(\boldsymbol{A}_{\boldsymbol{s}_{\mathbf{1}}} \boldsymbol{P}_{\boldsymbol{s}_{1}}\right)-P_{\mathrm{tot}, s_{1}}\right) \\
& -\mu_{2}\left(\operatorname{Tr}\left(\boldsymbol{A}_{\boldsymbol{s}_{\mathbf{2}}} \boldsymbol{P}_{\boldsymbol{s}_{\mathbf{2}}}\right)-P_{\mathrm{tot}, s_{2}}\right) \\
& -\nu\left(\operatorname{Tr}\left(\boldsymbol{B}_{\boldsymbol{s}_{\mathbf{1}}} \boldsymbol{P}_{\boldsymbol{s}_{1}}\right)+\operatorname{Tr}\left(\boldsymbol{B}_{\boldsymbol{s}_{\mathbf{2}}} \boldsymbol{P}_{\boldsymbol{s}_{\mathbf{2}}}\right)+\operatorname{Tr}\left(\boldsymbol{C}_{\boldsymbol{p}}\right)-P_{R}\right),
\end{aligned}
$$

where $\mu_{1}, \mu_{2}$, and $\nu$ are the positive Lagrange multipliers associated to the constraints (23), (24), and (25), respectively. By computing the derivative of $\mathcal{L}$ with respect to $P_{s_{i}}(j, j), i \in\{1,2\}, j \in\{1, \cdots, n\}$, and equating it to zero, we obtain the optimal power as follows

$$
P_{s_{i}}^{*}(j, j)=\left[\frac{1}{\mu_{i} A_{s_{i}}(j, j)+\nu B_{s_{i}}(j, j)}-Q_{\tilde{n}_{s_{i}}}(j, j)\right]^{+} .
$$

Equation (27) shows that the secondary transmission depends on the primary power allocation in addition to the available free eigenmodes since $P_{p}^{*}$ is included $\boldsymbol{Q}_{\tilde{n}_{s_{i}}}$ and $\overline{\boldsymbol{P}}_{p}$ is included in the matrices $\boldsymbol{A}_{\boldsymbol{s}_{i}}, \boldsymbol{B}_{\boldsymbol{s}_{i}}$, and $\boldsymbol{Q}_{\tilde{\boldsymbol{n}}_{s_{i}}}, i \in\{1,2\}$.

\section{Generalized Optimized Relay Gain}

In this section, we aim to further enhance the SU sum-rate by optimizing the relay gain. In the literature, the optimization of power and relay matrix or joint optimization was widely studied in the TWR context [29], [30]. In [16], it was shown that the optimal structure that maximizes the rate is given by the product of a two unitary matrices with a diagonal matrix. However, in our CR context, the structure of the amplification matrix $\boldsymbol{W}$ needs to follow the expression given in (17) in order to eliminate the effect of secondary interference in the free eigenmodes space of the PU. Note that this structure has a certain degree of freedom since $\overline{\boldsymbol{P}}_{\boldsymbol{w}}$ can have different structure as long as it belongs to $\mathcal{M}_{N_{p} ; N_{s} ; n_{p}}$. We study three cases of the matrix $\overline{\boldsymbol{P}}_{\boldsymbol{w}}$ depending on complexity-efficiency tradeoff: i) has the same value for the $n$ free eigenmodes, ii) $n$ values for the $n$ free eigenmodes, iii) $n$ full columns.

\section{A. Case I: $\overline{\boldsymbol{P}}_{\boldsymbol{w}}$ with Single Value Optimization}

Due to the complexity of finding the optimal entries of the matrix analytically, we assume in this case that the relay matrix is given by $\boldsymbol{W}=w\left(\boldsymbol{U}_{\boldsymbol{p}_{1}}^{\boldsymbol{H}} \boldsymbol{H}_{\boldsymbol{p}_{\boldsymbol{R}}}^{\boldsymbol{T}}\right)^{+} \overline{\boldsymbol{P}}_{\boldsymbol{p}}$ where the value of $w$ need to be optimized. In this case, the matrix $\boldsymbol{H}_{\boldsymbol{s}_{i}}^{\text {eqv }}$ can be re-written as follows:

$$
\boldsymbol{H}_{\boldsymbol{s}_{i}}^{\text {eqv }}=w \underbrace{\boldsymbol{H}_{\boldsymbol{s}_{i}}^{\boldsymbol{T}}\left(\boldsymbol{U}_{p_{1}}{ }^{H} \boldsymbol{H}_{p_{1} \boldsymbol{R}}\right)^{+} \boldsymbol{H}_{\boldsymbol{s}_{\bar{i}}} \boldsymbol{\Phi}_{s_{\bar{i}}}}_{\boldsymbol{H}_{s_{i} \mid w}^{\text {eqv }}} .
$$

In (28), we denote by $\boldsymbol{H}_{\boldsymbol{s}_{i} \mid \boldsymbol{w}}^{\text {eqv }}$ the component of $\boldsymbol{H}_{\boldsymbol{s}_{i}}^{\text {eqv }}$ that is not including the decision variable $w$. Thus, $\boldsymbol{\Psi}_{\boldsymbol{s}_{i}}$ can have the following expression

$$
\boldsymbol{\Psi}_{\boldsymbol{s}_{i}}=\frac{1}{w} \tilde{\boldsymbol{\Psi}}_{\boldsymbol{s}_{i}}=\frac{1}{w}\left(\tilde{\boldsymbol{H}}_{\boldsymbol{s}_{i} \mid \boldsymbol{w}}^{e q \boldsymbol{v}}\right)^{+}, i \in\{1,2\},
$$

where $\tilde{\boldsymbol{\Psi}}_{s_{i}}=\left(\tilde{\boldsymbol{H}}_{\boldsymbol{s}_{i} \mid \boldsymbol{w}}^{\text {eqv }}\right)^{+}$. Therefore, using (20), the noise covariance matrix can have the following form:

$$
\boldsymbol{Q}_{\tilde{n}_{s_{i}}}=\frac{1}{w^{2}} \boldsymbol{A}_{\boldsymbol{i}}+\boldsymbol{B}_{\boldsymbol{i}},
$$

where $\boldsymbol{A}_{\boldsymbol{i}}$ and $\boldsymbol{B}_{\boldsymbol{i}}$ are given in (31) and (32) at the top of the next page, respectively.

Similarly, the constraint (25), can be written as follows

$$
w^{2} \operatorname{Tr}\left(\boldsymbol{C}\left(\boldsymbol{P}_{\boldsymbol{s}_{\mathbf{1}}}, \boldsymbol{P}_{\boldsymbol{s}_{\mathbf{2}}}\right)\right) \leq P_{R},
$$

where $C$ is given in (33) also at the top of the next page. Hence, we convert the optimization problem expressed in (22)-(25) to the following one by including the new decision variable $w$ :

$$
\max _{\boldsymbol{P}_{\boldsymbol{s}_{1}}, \boldsymbol{P}_{\boldsymbol{P}_{\mathbf{2}}}, w} R_{\mathrm{tot}}=\sum_{i=1}^{2} \sum_{j=1}^{n} \log _{2}\left(1+\frac{P_{s_{i}}(j, j)}{\frac{1}{w^{2}} A_{i}(j, j)+B_{i}(j, j)}\right)
$$

$$
\text { s.t. (23), (24), (34). }
$$

Hence, the derivative of the Lagrangian function with respect to $w$ given $\boldsymbol{P}_{\boldsymbol{s}_{1}}$ and $\boldsymbol{P}_{\boldsymbol{s}_{2}}$ is given by

$$
\begin{gathered}
\frac{\partial \mathcal{L}}{\partial w}=2 w\left[\sum_{i=1}^{2} \sum_{j=1}^{n} \frac{A_{i, j} P_{s_{i}}(j, j)}{\left(A_{i, j}+w^{2} B_{i, j}\right)\left(A_{i, j}+w^{2}\left(P_{s_{i}}(j, j)+B_{i, j}\right)\right)}\right. \\
-\nu \operatorname{Tr}(\boldsymbol{C})],
\end{gathered}
$$

where $A_{i, j}=A_{i}(j, j)$ and $B_{i, j}=B_{i}(j, j)$. By equating the Lagrangian to zero, we obtain the following non-linear equation with one unknown variable:

$2 \nu \operatorname{Tr}(\boldsymbol{C})=\sum_{i=1}^{2} \sum_{j=1}^{n} \frac{A_{i, j} P_{s_{i}}(j, j)}{\left(A_{i, j}+w^{2} B_{i, j}\right)\left(A_{i, j}+w^{2}\left(P_{s_{i}}(j, j)+B_{i, j}\right)\right)}$.

We employ an iterative fixed-point algorithm in order to optimize SUs' transmitted powers and the amplification parameter $w$ as given in Algorithm 1. We start by initializing the values of $w$ and the Lagrangian multiplier $\nu$. Then, we determine the corresponding achieved rate using (27) (Line 3). Then, the algorithm updates the value of $w$ using the powers obtained in Line 3. This approach is repeated till achieving convergence, i.e., when the achieved data rate remains unchanged as indicated in Line 11 of Algorithm 1. The value of $\nu$ is updated using a typical backtracking line search method [36].

Note that we do not claim the convergence to the global maximum of the problem similarly to [37]. However, in the numerical results, we use multiple initial values (Line 2) to prevent the convergence to local maxima. We found that they converge to the same secondary sum-rate. That is the algorithm converges to a unique solution and is not trapped in different local maxima. Hence, the reached solution can correspond to the global maximum of the problem (See Appendix A form more details).

\section{B. Case II: $\overline{\boldsymbol{P}}_{\boldsymbol{w}}$ with $n$ Values Optimization}

In this case, $\overline{\boldsymbol{P}}_{\boldsymbol{w}}$ has $n$ values to be optimized. In fact for $j=1, \ldots, \min \left\{N_{p}, N_{s}\right\}$ we have $\bar{P}_{w}(j, j)=w_{j}$, if $P_{p}^{*}(j, j)=0$, and $\bar{P}_{w}(j, j)=0$, otherwise. Determining the values of $w_{j}$ analytically as a function of the power similarly to (37) is difficult and involves $n$ nonlinear equations. Therefore, in our simulation results section (Section VI), we numerically investigate the performance of the diagonal matrix 


$$
\begin{aligned}
& \boldsymbol{A}_{\boldsymbol{i}}=\tilde{\boldsymbol{\Psi}}_{s_{i}} \boldsymbol{H}_{\boldsymbol{s}_{i} p_{2}} \boldsymbol{V}_{p_{1}} \boldsymbol{P}_{p}^{*}\left(\tilde{\boldsymbol{\Psi}}_{s_{i}} \boldsymbol{H}_{s_{i} p_{2}} \boldsymbol{V}_{\boldsymbol{p}_{1}}\right)^{H}+\tilde{\Psi}_{s_{i}} \tilde{\Psi}_{s_{i}}^{\boldsymbol{H}} \\
& \boldsymbol{B}_{i}=\tilde{\Psi}_{s_{i}} \boldsymbol{H}_{s_{i}}\left(\boldsymbol{U}_{p_{1}}^{H} H_{p_{1} R}\right)^{+} \boldsymbol{H}_{p_{1} R} V_{p_{1}} P_{p}^{*}\left(\tilde{\Psi}_{s_{i}} H_{s_{i}}\left(U_{p_{1}}^{H} H_{p_{1} R}\right)^{+} H_{p_{1} R} V_{p_{1}}\right)^{H} \\
& +\tilde{\Psi}_{s_{i}} \boldsymbol{H}_{s_{i}}\left(\boldsymbol{U}_{p_{1}}^{H} \boldsymbol{H}_{p_{1} R}\right)^{+}\left(\tilde{\Psi}_{s_{i}} \boldsymbol{H}_{s_{i}}\left(\boldsymbol{U}_{p_{1}}^{H} \boldsymbol{H}_{p_{1} R}\right)^{+}\right)^{H}, \\
& \boldsymbol{C}=\left(\left(\boldsymbol{U}_{\boldsymbol{p}_{1}}^{\boldsymbol{H}} \boldsymbol{H}_{\boldsymbol{p}_{1} \boldsymbol{R}}\right)^{+} \boldsymbol{H}_{\boldsymbol{s}_{1}} \boldsymbol{\Phi}_{\boldsymbol{s}_{1}} \boldsymbol{P}_{\boldsymbol{s}_{1}}\left(\left(\boldsymbol{U}_{\boldsymbol{p}_{1}}^{\boldsymbol{H}} \boldsymbol{H}_{\boldsymbol{p}_{1} \boldsymbol{R}}\right)^{+} \boldsymbol{H}_{\boldsymbol{s}_{1}} \boldsymbol{\Phi}_{\boldsymbol{s}_{1}}\right)^{H}\right. \\
& +\left(\boldsymbol{U}_{\boldsymbol{p}_{1}}^{\boldsymbol{H}} \boldsymbol{H}_{\boldsymbol{p}_{1} \boldsymbol{R}}\right)^{+} \boldsymbol{H}_{\boldsymbol{s}_{\mathbf{2}}} \boldsymbol{\Phi}_{\boldsymbol{s}_{\mathbf{2}}} \boldsymbol{P}_{\boldsymbol{s}_{\mathbf{2}}}\left(\left(\boldsymbol{U}_{\boldsymbol{p}_{1}}^{\boldsymbol{H}} \boldsymbol{H}_{\boldsymbol{p}_{1} \boldsymbol{R}}\right)^{+} \boldsymbol{H}_{\boldsymbol{s}_{\mathbf{2}}} \boldsymbol{\Phi}_{\boldsymbol{s}_{\mathbf{2}}}\right)^{H} \\
& \left.+\left(\boldsymbol{U}_{\boldsymbol{p}_{1}}^{\boldsymbol{H}} \boldsymbol{H}_{p_{1} \boldsymbol{R}}\right)^{+} \boldsymbol{H}_{\boldsymbol{p}_{1} \boldsymbol{R}} \boldsymbol{V}_{\boldsymbol{p}_{1}} \boldsymbol{P}_{\boldsymbol{p}}^{*}\left(\left(\boldsymbol{U}_{\boldsymbol{p}_{1}}^{\boldsymbol{H}} \boldsymbol{H}_{p_{1} \boldsymbol{R}}\right)^{+} \boldsymbol{H}_{\boldsymbol{p}_{1} \boldsymbol{R}} \boldsymbol{V}_{\boldsymbol{p}_{1}}\right)^{H}+\left(\boldsymbol{U}_{\boldsymbol{p}_{1}}^{\boldsymbol{H}} \boldsymbol{H}_{\boldsymbol{p}_{1} \boldsymbol{R}}\right)^{+}\left(\boldsymbol{U}_{\boldsymbol{p}_{1}}^{\boldsymbol{H}} \boldsymbol{H}_{p_{1} \boldsymbol{R}}\right)^{+}{ }^{H}\right) .
\end{aligned}
$$

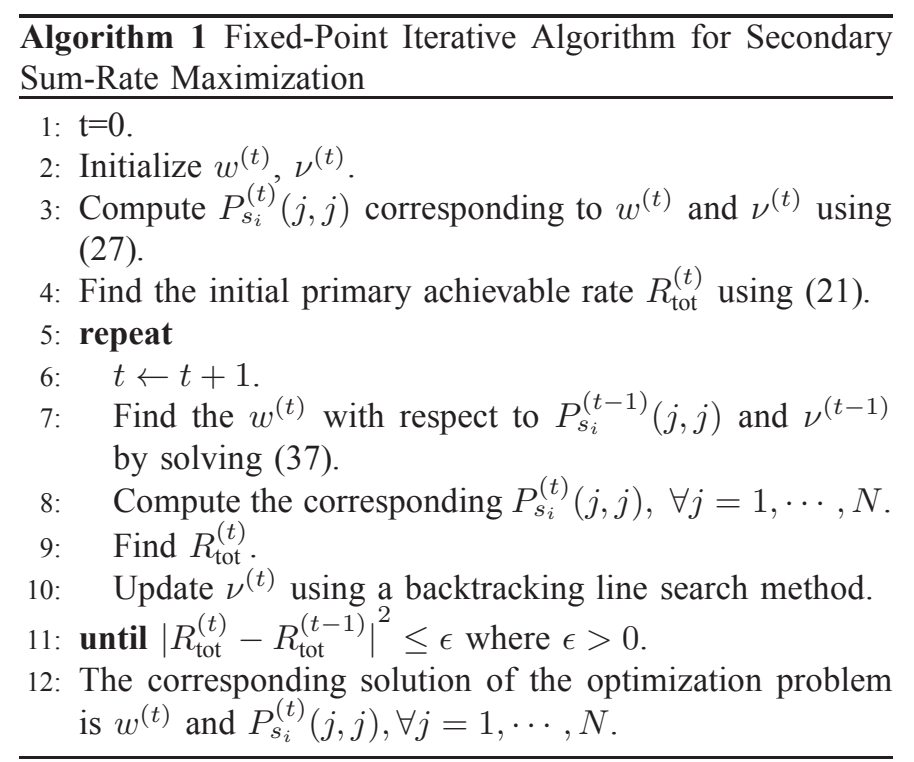

and compare it with other cases. Certainly, the increase of the number of parameters in $\overline{\boldsymbol{P}}_{\boldsymbol{w}}$ enhances the system performance. However, this secondary sum-rate enhancement will be obtained at the cost of higher computational complexity. Yet the choice of a diagonal matrix remains with a lower complexity than the more general scenario where $\overline{\boldsymbol{P}}_{\boldsymbol{w}}$ is a full matrix considered in Case III.

\section{Case III: $\overline{\boldsymbol{P}}_{\boldsymbol{w}}$ with n Full Columns Optimization}

In the most general case, $\overline{\boldsymbol{P}}_{\boldsymbol{w}}$ is a full matrix belonging to $\mathcal{M}_{N_{p} ; N_{s} ; n}$ which means that $\overline{\boldsymbol{P}}_{\boldsymbol{w}}$ has $n$ full columns. Hence, by following (17), the number of parameters in $W$ to be determined is $N_{s} \times n$ for entries. Theoretically, optimizing this structure of $\overline{\boldsymbol{P}}_{\boldsymbol{w}}$ will achieve the highest secondary performance. A comparison between the three cases is provided in Section VI. Case II is considered here in order to achieve a tradeoff between the computational complexity and the best achieved performance using the full matrix case. Note that the optimization of the general structure of $\mathrm{W}$ is performed numerically, i.e., using the interior-point algorithm implemented in the Matlab optimization tools. The purpose of presenting this case is to show the upper limit of the performances compared to case I and case II.

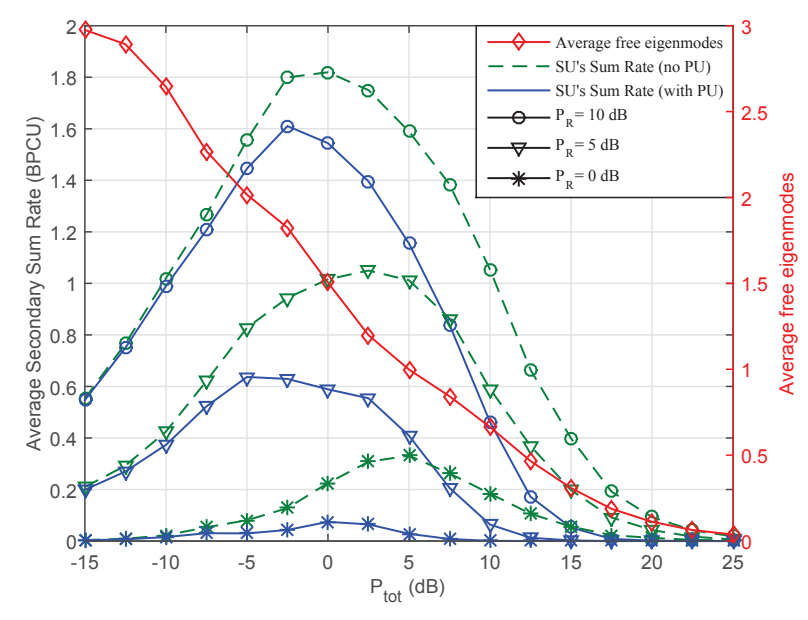

Figure 2: SUs sum-rate as a function of $P_{\text {tot }}$ with $w=1$.

\section{Numerical Results}

In our numerical results, we consider a Rayleigh fading channel in which the channel gains are complex Gaussian random variables with zero mean and unit variance. The rates are expressed in bits per channel use (BPCU). We use the same budget power for all the users, i.e., $P_{\mathrm{tot}, s_{1}}=P_{\mathrm{tot}, s_{2}}=$ $P_{\text {tot }, p_{1}}=P_{\text {tot }, p_{2}}=P_{\text {tot }}$. The primary and secondary systems are also equipped with the same number of antennas, i.e., $N_{p}=N_{s}=N$. In the rest of the figures except Fig. 5, we take $N=4$ antennas.

In Fig. 2, we plot the average SUs sum-rate as a function of $P_{\text {tot }}$ with different values of the relay power, $P_{R}=0,5,10 \mathrm{~dB}$. We compare between the case when the PU is present and the SU interference is limited to the free eigenmodes, and the case when the PU is absent, i.e., "no PU". In this latter case, the SUs and the relay still use the same precoding choice, even if there is no primary transmission, i.e., $\overline{\boldsymbol{P}}_{\boldsymbol{p}}=\boldsymbol{I}_{\boldsymbol{N}}$, in order to have a fair comparison between the two cases. Hence, we notice that the TWR SA offers a positive rate gain for the SUs. At low values of $P_{\text {tot }}$, the rate gain is almost equal to the "no PU" rates due to the availability of the free eigenmodes. In mid-range power, i.e., between -5 and $5 \mathrm{~dB}$, this gain attains a maximum which presents $33-85 \%$ of the SU "no PU" sum-rate depending on the relay power $P_{R}$. This remarkable gain is explained by the fact that the $\mathrm{SU}$ 


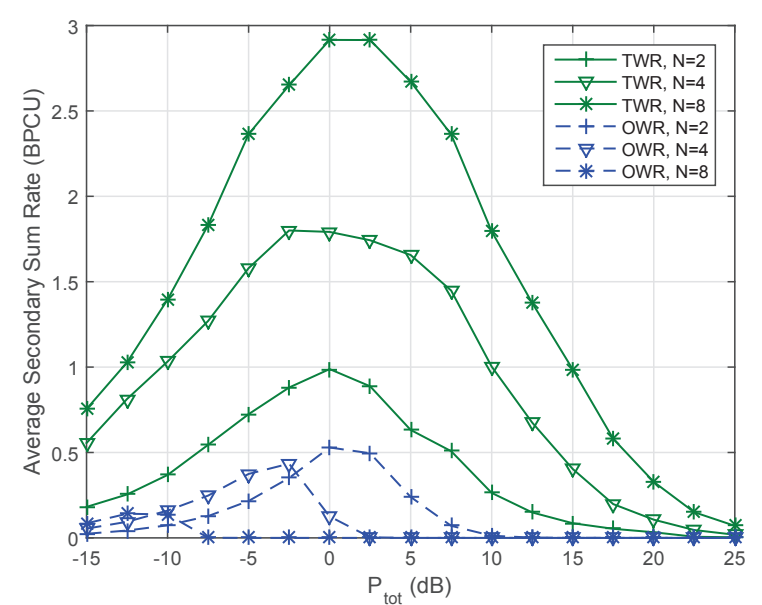

Figure 3: TWR and OWR SUs sum-rate as a function of $P_{\text {tot }}$ with $w=1$.

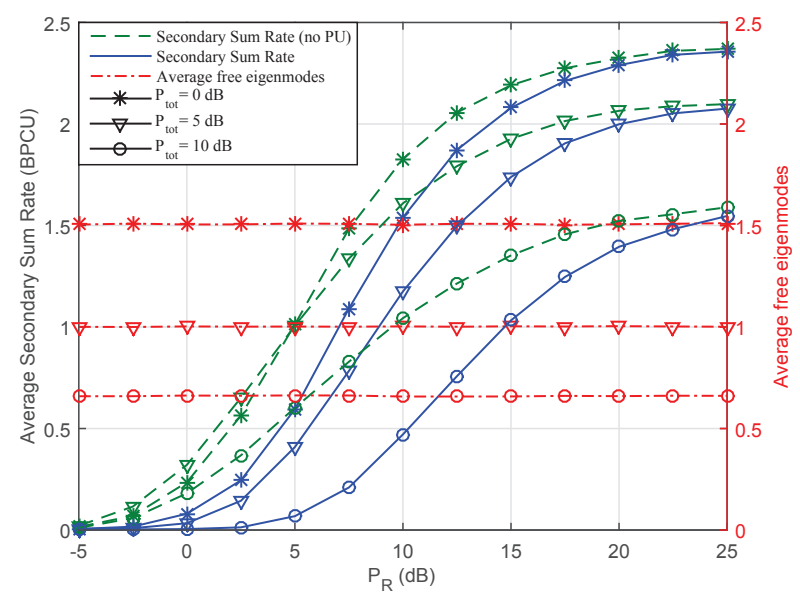

Figure 4: SU sum-rate vs. $P_{R}$ with various $P_{\text {tot }}$ and $w=1$.

power is high and there are still some free eigenmodes (red curve) to be exploited. However, when $P_{\text {tot }}$ has large values, the free eigenmodes (in the red curve) become very limited and with the high interference coming from the PUs, SU sumrate present relatively low values. Hence, there is an optimal value of $P_{\text {tot }}$ that allows the SUs to reach a maximum rate gain without affecting the PU performance.

In Fig. 3, we plot the TWR and OWR rates with different antennas number $N$. We found that that TWR provides better performances than the OWR at low power budget values. Although OWR can avoid the self-interference, TWR performs transmission in two time slots instead of four. Moreover, cancelling the self-interference in TWR is not a complex process since the sources can estimate the channels and knows their own transmitted signal. Also, it should be noted that the interference cancellation due to the SA precoding is needed for both OWR and TWR.

In Fig. 4, the SU sum-rate is plotted as a function of the relay power, $P_{R}$ to highlight its effect on the cognitive

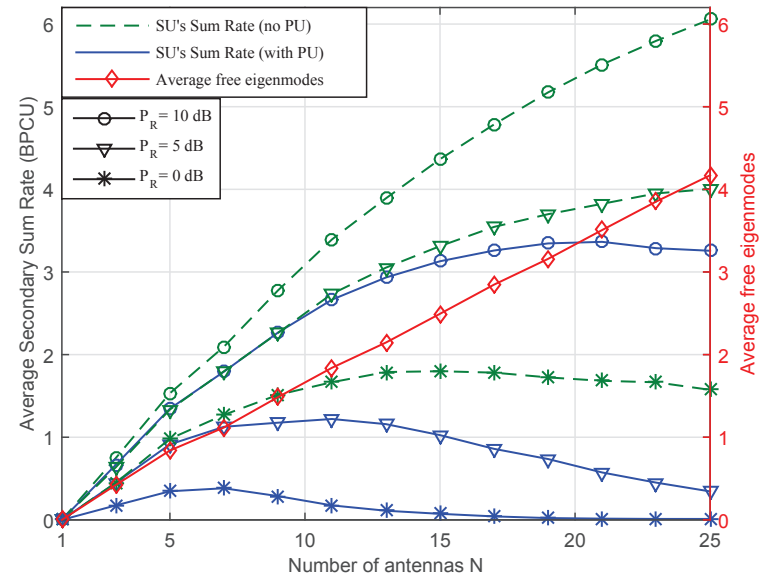

Figure 5: SUs sum-rate vs. $P_{\text {tot }}$ with various $P_{R}$, $P_{t o t}=10 \mathrm{~dB}$ and $w=3$.

performance. Recall that the average number of free eigenmodes remains the same since it only depends on the PU power allocation. We notice that as $P_{\text {tot }}$ increases, the $\mathrm{SU}$ sum-rate decreases. This is mainly caused by the fact that as $P_{\text {tot }}$ increases, the PUs are able to transmit with higher power using more eigenmodes which results in both high primary to secondary interference and lower number of free eigenmodes (as shown by the red curves). From another side, we show that in order to achieve acceptable SU sum-rate, the relay power should be greater than $0 \mathrm{~dB}$ otherwise the resulting rate is very low $(<0.1 \mathrm{BPCU})$. In addition, as $P_{R}$ increases, the SU sum-rate in presence of PUs approaches the rate with no PUs. Meaning that the ratio of the SU SNR over the interference from the PUs is increasing with $P_{R}$ allowing to achieve rates close the no PUs case.

In Fig. 5, we plot the SU sum-rate as a function of the number of antennas, $N$, to highlight its effect on the TWR cognitive performance. We first show that the variation of the number of free eigenmodes is linear with the number of antennas. In this setting, where $P_{\text {tot }}=10 \mathrm{~dB}$, the number of free eigenmodes is about $\frac{N}{6}$. However, depending on $P_{R}$ there is an optimal number of antennas that gives a maximum sum-rate and if the relay selects this same number even if $N$ increases, the sum-rate will remain at its maximum value. Afterwards, when $\mathrm{N}$ increases the SU sum-rate decreases due to the additional PUs interference caused by the higher number of antennas.

In Fig. 6, the effect of the relay amplification matrix gain $W$ on the SU sum-rate is analyzed. This figure highlights the case where $W=w \times\left(\boldsymbol{U}_{\boldsymbol{p}_{1}}{ }^{H} \boldsymbol{H}_{\boldsymbol{p}_{1} \boldsymbol{R}}\right)^{+} \overline{\boldsymbol{P}}_{\boldsymbol{w}}$, which is not necessarily the optimal choice but is a simple one to quantify the effect of this matrix on the system performance. We, first, note that, for $N=4$, the average free eigenmodes is 1 , whereas the achievable sum-rate is about $75 \%$ of the "no PU" case that can exploit all the 4 antennas freely. This means that the TWR is three times efficient in a CR context than in single communication case with SA precoding. Consequently, TWR is a promising technique for MIMO CR paradigm. From 


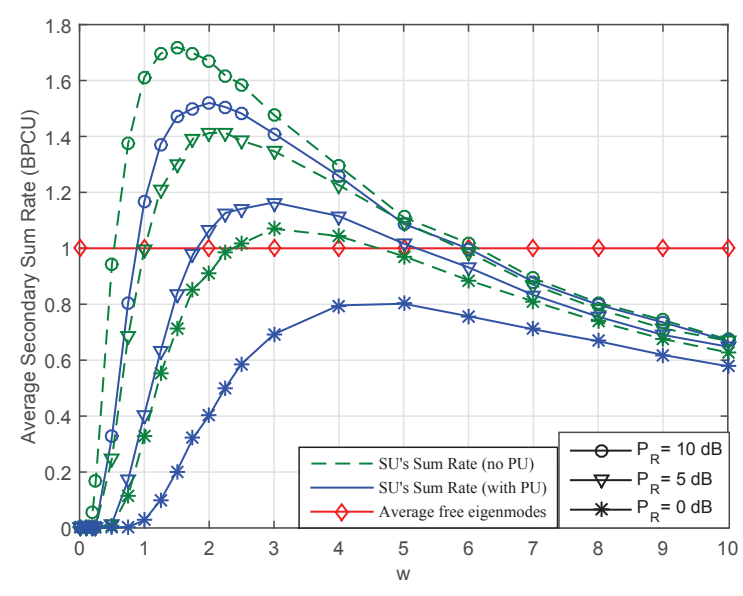

Figure 6: SUs sum-rate as a function of $w$ with various values of $P_{R}$ and $P_{t o t}=5 \mathrm{~dB}$.

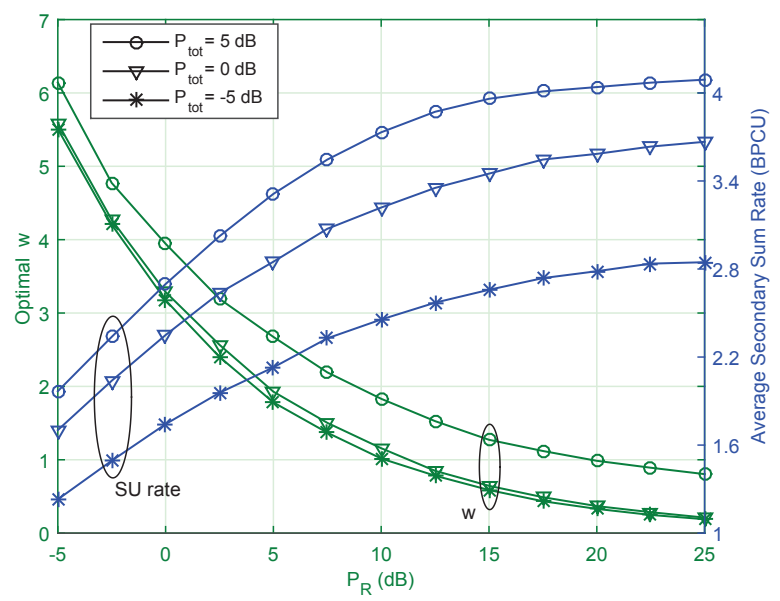

Figure 7: Optimized $w$ SUs sum-rate as a function of $P_{R}$ with various values of $P_{\text {tot }}$.

another side, there is an optimal value of $w$ that maximizes the SU sum-rate. This value should be adapted with $P_{R}$ in order to have an efficient use of the relay. We also show that as $P_{R}$ increases the maximum SU sum-rate increases and the optimal $w$ decreases.

In Fig. 7, we highlight the performance of the optimized relay matrix with case I, i.e., $\boldsymbol{W}=w \times\left(\boldsymbol{U}_{p_{1}}^{H} \boldsymbol{H}_{p_{1} \boldsymbol{R}}\right)^{+} \overline{\boldsymbol{P}}_{\boldsymbol{w}}$. We plot the resulting sum-rate and average $w$ as a function of the relay power for different values of $P_{\text {tot }}$ with averaging on different channel realizations. We also assume that the PU budget power in this case, is fixed and equal to $0 \mathrm{~dB}$. We show that $w$ is a convex-decreasing function of $P_{R}$ and that $w \rightarrow 0$ when $P_{R} \rightarrow \infty$. This observation could be explained by the fact that when the relay power $P_{R}$ is small, the transmitted signal should be further amplified in order to maximize the sum-rate. However, when $P_{R}$ is high, the amplification should be reduced since it affects the resulting SNR when the interference from the PU and the noise are

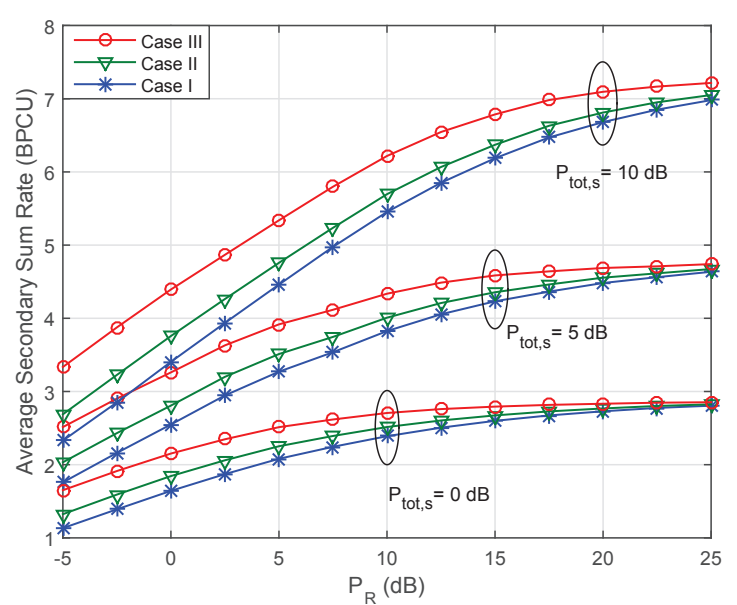

Figure 8: Sum-rate as a function of $P_{R}$ with various relay matrix structures with $P_{t o t, p}=0 \mathrm{~dB}$.

amplified. We also show that the sum-rate is higher than the maximum rate achieved in Fig. 6. In fact, for each channel realization in our proposed algorithm, the corresponding power and $w$ solutions are computed accordingly. However, in Fig. 6, the value of $w$ is fixed for all realizations which affects the overall sum-rate.

In Fig. 8, we highlight the effect of the amplification matrix structure by plotting the sum-rate as a function of $P_{R}$ for the three cases with different values of $P_{\text {tot }}$ where the values of the matrix $\boldsymbol{W}$ are determined numerically. We show that in general, the gain in sum-rate between case I and case II is relatively small. For instance, for $P_{R}=5 \mathrm{~dB}$ this gain is about $8 \%$ for $P_{t o t, s}=0 \mathrm{~dB}$ and $6 \%$ for $P_{t o t, s}=10 \mathrm{~dB}$. Meanwhile, the rate gain between case I and case III is $20 \%$ for both $P_{t o t, s}=0$ and $10 \mathrm{~dB}$ which means that the scalar model achieves $80 \%$ of the rate that the full matrix archives. Hence, there is a slight trade-off between complexity and rate. In addition, we show that as $P_{R}$ increases the performances of the three cases converge to the same value. Consequently, there is no need to model the amplification gain as a full matrix since the scalar model, i.e., case I, achieves almost the same rate of case III mainly for high $P_{R}$.

In Fig. 9, we present a complexity study of Algorithm 1 with the increasing number of antennas $N$. We run our algorithm on an Intel $2.67 \mathrm{GHz}$ (2 processors) workstation running on Windows. We then perform a curve fitting to the obtained curve and we found that the complexity of our algorithm is $O\left(N^{3}\right)$.

\section{CONCLUSION}

In this paper, we studied a cognitive amplify-and-forward (AF) two-way relaying (TWR) scheme with space alignment to protect the primary user (PU). We determined the secondary precoders structures that avoid interfering with the PU. We also compute the power allocation that maximizes the PU rate and the cognitive sum-rate. We presented a joint optimization algorithm that considers optimizing the relay gain matrix using 


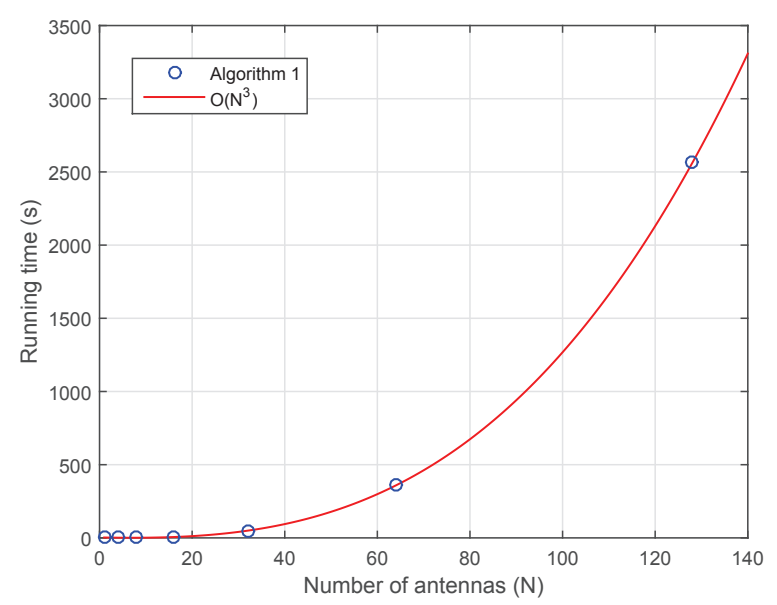

Figure 9: Running time of Algorithm 1 vs. number of antennas $N$.

three models depending on the gain matrix structure complexity. In our numerical results, we investigated the sum-rate achieved using TWR with SA for different system parameters such as the relay power, gain matrix and the number of antennas. We also highlighted how does the relay gain affect the secondary sum-rate. Finally, we compare the performances of the different gain matrix structures and showed that a simple structure achieves up to $80 \%$ of the optimal structure.

\section{ACKNOWLEDGEMENT}

This work was supported by the Qatar National Research Fund (a member of Qatar Foundation) under NPRP Grant NPRP 6-001-2-001. The statements made herein are solely the responsibility of the authors

\section{Appendix A : Analysis of the convergence of Algorithm 1}

In each step, the algorithm optimizes the objective function with respect to $w$ with fixed powers (line 7) then with respect to the powers with fixed $w$ (line 8 ) in the form of a fixed-point algorithm.

i) From one side, when $w$ is fixed it is clear that the objective function $R_{\text {tot }}$ is convex with respect to the powers as we showed in Sections III and IV. Hence, the obtained powers are optimal for this given value of $w$.

ii) From the other side, when the powers are fixed, we have

1) $R_{\text {tot }} \geq 0 \forall w$

2) $\lim _{w \rightarrow 0} R_{\text {tot }}=0$

3) $\lim _{w \rightarrow \infty} R_{\text {tot }}=0$. Note that 3 ) comes form the fact that infinite amplification forces the transmit powers to be 0 in order to avoid infringing the relay power constraint in (25) when amplifying the noise, i.e., the term $\boldsymbol{W} \boldsymbol{W}^{H}$.

4) $R_{\text {tot }}$ is not multimodal with respect to $w$ since from (35) $R_{\text {tot }}$ is a combination of a logarithmic function and a quadratic function of $w$.

Hence, $R_{\text {tot }}$ has a global maxima with respect to $w$ and the corresponding $w$ is optimal for the given powers.
From another side, at each iteration, we have $R_{\text {tot }}^{(t+1)} \geq R_{\text {tot }}^{(t)}$ since we alternatively maximize $R_{t o t}$ in line 7 and line 8 meaning that $R_{\text {tot }}$ is increasing in each iteration of the algorithm.

\section{REFERENCES}

[1] L. Sboui, H. Ghazzai, Z. Rezki, and M.-S. Alouini, "On achievable rate of two-way relaying cognitive radio with space alignment," in Proc. of the IEEE International Conference on Communications (ICC'15), London, UK, June 2015.

[2] "Spectrum policy task force," Federal Communications Commission, Tech. Rep. ET Docket no. 02-135, Nov. 2002.

[3] J. Mitola and G. Q. Maguire, "Cognitive radio: Making software radios more personal," IEEE. Personal Communications, vol. 6, no. 4, pp. 1318, Aug. 1999.

[4] J. Mitola, "Cognitive Radio: An Integrated Agent Architecture for Software Defined Radio," Ph.D. dissertation, Royal Institute of Technology (KTH), Stockholm, Sweden, May 2000.

[5] S. Haykin, "Cognitive radio: Brain-empowered wireless communications," IEEE Journal on Selected Areas in Communications, vol. 23, no. 2, pp. 201-220, Feb. 2005.

[6] E. Telatar, "Capacity of multi-antenna Gaussian channels," European Transactions on Telecommunications, vol. 10, no. 6, pp. 585-595, Dec. 1999.

[7] S. Perlaza, M. Debbah, S. Lasaulce, and J.-M. Chaufray, "Opportunistic interference alignment in MIMO interference channels," in Proc. of the 19th IEEE International Symposium on Personal, Indoor and Mobile Radio Communications (PIMRC'08), Cannes, France, Sept. 2008.

[8] L. Sboui, H. Ghazzai, Z. Rezki, and M.-S. Alouini, "Achievable rate of cognitive radio spectrum sharing MIMO channel with space alignment and interference temperature precoding," in Proc. of the IEEE International Conference on Communications (ICC'13), Budapest, Hungary, June 2013.

[9] L. Sboui, H. Ghazzai, Z. Rezki, and M. Alouini, "Achievable rate of spectrum sharing cognitive radio multiple-antenna channels," IEEE Transactions on Wireless Communications, vol. 9, no. 14, pp. 48474856, Jan. 2015.

[10] A. Alizadeh, H. R. Bahrami, M. Maleki, and S. Sastry, "Spatial sensing and cognitive radio communication in the presence of a $\mathrm{K}$ user interference primary network," IEEE Journal on Selected Areas in Communications, vol. 33, no. 5, pp. 741-754, May 2015.

[11] L. Sboui, H. Ghazzai, Z. Rezki, and M. S. Alouini, "Achievable rate of a cognitive mimo multiple access channel with multi-secondary users," IEEE Communications Letters, vol. 19, no. 3, pp. 403-406, March 2015.

[12] D. Gunduz, A. Yener, A. Goldsmith, and H. Poor, "The multi-way relay channel," in Proc. International Symposium on Information Theory (ISIT'09), Seoul, Korea, June-July 2009.

[13] B. Wang, J. Zhang, and A. Host-Madsen, "On the capacity of MIMO relay channels," IEEE Transactions on Information Theory, vol. 51, no. 1, pp. 29-43, Jan. 2005.

[14] T. Luan, F. Gao, X.-D. Zhang, J. Li, and M. Lei, "Robust beamforming for relay-aided multiuser MIMO cognitive radio networks," in Proc. of the IEEE Wireless Communications and Networking Conference (WCNC'13), Shanghai, China, Apr. 2013.

[15] M. Hasna and M.-S. Alouini, "Optimal power allocation for relayed transmissions over Rayleigh-fading channels," IEEE Transactions on Wireless Communications, vol. 3, no. 6, pp. 1999-2004, Nov. 2004.

[16] L. Sanguinetti, A. D'Amico, and Y. Rong, "A tutorial on the optimization of amplify-and-forward MIMO relay systems," IEEE Journal on Selected Areas in Communications, vol. 30, no. 8, pp. 1331-1346, Sept. 2012.

[17] T. Wang, A. Cano, G. Giannakis, and J. Laneman, "High-performance cooperative demodulation with decode-and-forward relays," IEEE Transactions on Communications, vol. 55, no. 7, pp. 1427-1438, July 2007.

[18] G. Kramer, M. Gastpar, and P. Gupta, "Cooperative strategies and capacity theorems for relay networks," IEEE Transactions on Information Theory, vol. 51, no. 9, pp. 3037-3063, Sept. 2005.

[19] L. Sboui, H. Ghazzai, Z. Rezki, and M.-S. Alouini, "On the throughput of a relay-assisted cognitive radio MIMO channel with space alignment," in 12th International Symposium and Workshops on Modeling and Optimization in Mobile, Ad Hoc and Wireless Networks (WiOpt '14), Hammamet, Tunisia, May 2014. 
[20] A. Alsharoa, H. Ghazzai, and M.-S. Alouini, "Near-optimal power allocation with PSO algorithm for MIMO cognitive networks using multiple AF two-way relays," in IEEE International Conference on Communications (ICC'14), Sydney, Australia, June 2014.

[21] G. Wang, Y. Zou, J. Lu, and C. Tellambura, "Cognitive transmission and performance analysis for amplify-and-forward two-way relay networks," in IEEE International Conference on Communications (ICC'14), Sydney, Australia, June, 2014

[22] M. Hafeez and J. Elmirghani, "Analysis of dynamic spectrum leasing for coded bi-directional communication," IEEE Journal on Selected Areas in Communications, vol. 30, no. 8, pp. 1500-1512, Sept. 2012.

[23] Z. Ding, I. Krikidis, B. Rong, J. Thompson, C. Wang, and S. Yang, "On combating the half-duplex constraint in modern cooperative networks: protocols and techniques," IEEE Transactions on Wireless Communications, vol. 19, no. 6, pp. 20-27, Dec. 2012.

[24] M. Eslamifar, W. H. Chin, C. Yuen, and Y. L. Guan, "Performance analysis of two-step bi-directional relaying with multiple antennas," IEEE Transactions on Wireless Communications, vol. 11, no. 12, pp. 4237-4242, Dec. 2012.

[25] A. Alizadeh, S.-S. Sadough, and N. Khajavi, "Optimal beamforming in cognitive two-way relay networks," in IEEE 21st International Symposium on Personal Indoor and Mobile Radio Communications (PIMRC'10), Istanbul, Turkey, Sept. 2010.

[26] K. Jitvanichphaibool, Y.-C. Liang, and R. Zhang, "Beamforming and power control for multi-antenna cognitive two-way relaying," in IEEE Wireless Communications and Networking Conference (WCNC'09), Budabest, Hungary, Apr. 2009.

[27] R. Wang, M. Tao, and Y. Liu, "Optimal linear transceiver designs for cognitive two-way relay networks," IEEE Transactions on Signal Processing, vol. 61, no. 4, pp. 992-1005, Feb. 2013.

[28] Q. Li, S. H. Ting, A. Pandharipande, and Y. Han, "Cognitive spectrum sharing with two-way relaying systems," IEEE Transactions on Vehicular Technology, vol. 60, no. 3, pp. 1233-1240, Mar. 2011.

[29] R. Wang and M. Tao, "Joint source and relay precoding designs for MIMO two-way relaying based on MSE criterion," IEEE Transactions on Signal Processing, vol. 60, no. 3, pp. 1352-1365, Mar. 2012.

[30] S. Xu and Y. Hua, "Optimal design of spatial source-and-relay matrices for a non-regenerative two-way MIMO relay system," IEEE Transactions on Wireless Communications, vol. 10, no. 5, pp. 1645-1655, May 2011.

[31] R. Zhang, Y.-C. Liang, C. C. Chai, and S. Cui, "Optimal beamforming for two-way multi-antenna relay channel with analogue network coding," IEEE Journal on Selected Areas in Communications, vol. 27, no. 5, pp. 699-712, June 2009.

[32] P. Ubaidulla and S. Aissa, "Robust two-way cognitive relaying: Precoder designs under interference constraints and imperfect CSI," IEEE Transactions on Wireless Communications, vol. 13, no. 5, pp. 2478-2489, May 2014.

[33] G. Wang, Q. Liu, R. He, F. Gao, and C. Tellambura, "Acquisition of channel state information in heterogeneous cloud radio access networks: challenges and research directions," IEEE Wireless Communications, vol. 22, no. 3, pp. 100-107, June 2015.

[34] A. Nasir, H. Mehrpouyan, S. Durrani, S. D. Blostein, R. Kennedy et al., "Training-based synchronization and channel estimation in AF two-way relaying networks," in IEEE 15th International Workshop on Signal Processing Advances in Wireless Communications (SPAWC'14), Toronto, Canada, June 2014.

[35] S. Boyd and L. Vandenberghe, Convex Optimization. Cambridge University Press, 2004.

[36] L. Armijo et al., "Minimization of functions having lipschitz continuous first partial derivatives," Pacific Journal of mathematics, vol. 16, no. 1, pp. 1-3, 1966.

[37] L. Lin, R. D. Yates, and P. Spasojevic, "Adaptive transmission with discrete code rates and power levels," IEEE Transactions on Communications, vol. 51, no. 12, pp. 2115-2125, Dec. 2003.

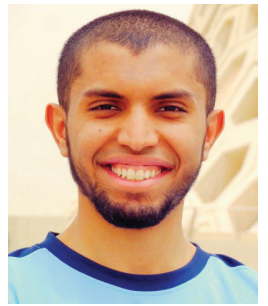

Lokman Sboui (S'11) was born in Cairo, Egypt. He received the Diplome d'Ingénieur degree with honors from École Polytechnique de Tunisie (EPT), La Marsa, Tunisia, in 2011, the Master degree from King Abdullah University of Science and Technology (KAUST) in May 2013. He is currently a Ph.D. candidate in the Electrical Engineering program of KAUST. His current research interests include: performance of cognitive radio systems, low SNR communication, MIMO communication, relaying performances, energy efficient power allocation and green

wireless sensor networks.

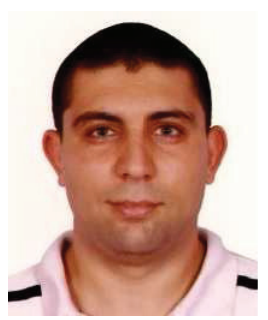

Hakim Ghazzai (S'12, M'15) was born in Tunisia $\mathrm{He}$ is currently working as a research scientist at Qatar Mobility Innovations Center (QMIC), Doha, Qatar. He received his $\mathrm{PhD}$ degree in Electrical Engineering from King Abdullah University of Science and Technology (KAUST), Saudi Arabia in 2015. He received his Diplome d'Ingenieur in telecommunication engineering and Master of Science degree in high-rate transmissions systems from the Ecole $\mathrm{Su}$ perieure des Communications de Tunis (SUP'COM), Tunis, Tunisia in 2010 and 2011, respectively. His general research interests include mobile and wireless networks, green communications, internet of things, and optimization.

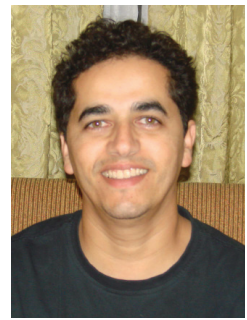

Zouheir Rezki (S'01-M'08-SM'13) was born in Casablanca, Morocco. He received the Diplôme d'Ingénieur degree from the École Nationale de l'Industrie Minérale (ENIM), Rabat, Morocco, in 1994, the M.Eng. degree from École de Technologie Supérieure, Montreal, Québec, Canada, in 2003, and the Ph.D. degree from École Polytechnique, Montreal, Québec, in 2008, all in electrical engineering. From October 2008 to September 2009, he was a postdoctoral research fellow with Data Communications Group, Department of Electrical and Computer Engineering, University of British Columbia. Then, has been a Senior Research Scientist at King Abdullah University of Science and Technology (KAUST), Saudi Arabia until June 2016. He joined University of Idaho as a Faculty Member on August 2016. His research interests include: performance limits of communication systems, physical-layer security, cognitive and sensor networks and low-complexity detection algorithms.

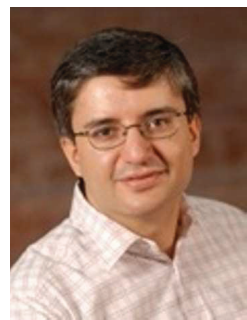

Mohamed-Slim Alouini (S'94, M'98, SM'03, F09) was born in Tunis, Tunisia. He received the Ph.D. degree in Electrical Engineering from the California Institute of Technology (Caltech), Pasadena, CA, USA, in 1998. He served as a faculty member in the University of Minnesota, Minneapolis, MN, USA, then in the Texas A\&M University at Qatar, Education City, Doha, Qatar before joining King Abdullah University of Science and Technology (KAUST), Thuwal, Makkah Province, Saudi Arabia as a Professor of Electrical Engineering in 2009. His current research interests include the modeling, design, and performance analysis of wireless communication systems. 